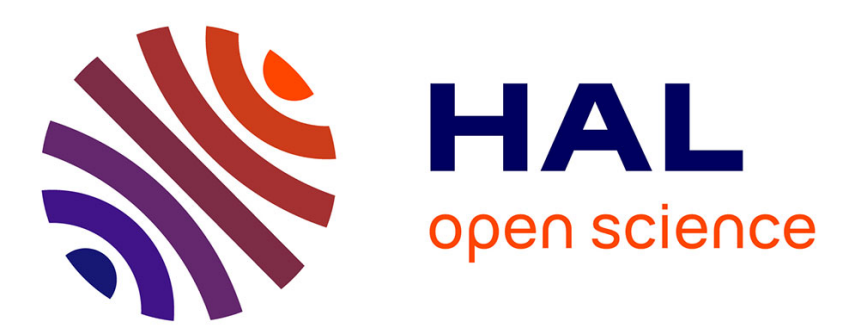

\title{
Effects of nonlinear wave coupling: Accelerated solitons
}

\author{
Antonio Degasperis, Matteo Conforti, Fabio Baronio, Stefan Wabnitz
}

\section{To cite this version:}

Antonio Degasperis, Matteo Conforti, Fabio Baronio, Stefan Wabnitz. Effects of nonlinear wave coupling: Accelerated solitons: Boomerons: From curiosity to nonlinear optics. The European Physical Journal. Special Topics, 2007, 147 (1), pp.233-252. 10.1140/epjst/e2007-00211-y · hal-02405213

\section{HAL Id: hal-02405213 \\ https://hal.science/hal-02405213}

Submitted on 11 Dec 2019

HAL is a multi-disciplinary open access archive for the deposit and dissemination of scientific research documents, whether they are published or not. The documents may come from teaching and research institutions in France or abroad, or from public or private research centers.
L'archive ouverte pluridisciplinaire HAL, est destinée au dépôt et à la diffusion de documents scientifiques de niveau recherche, publiés ou non, émanant des établissements d'enseignement et de recherche français ou étrangers, des laboratoires publics ou privés. 


\title{
Effects of nonlinear wave coupling: accelerated solitons
}

\section{Boomerons: from curiosity to nonlinear optics}

Antonio Degasperis ${ }^{1, a}$, Matteo Conforti ${ }^{2}$, Fabio Baronio ${ }^{2}$, and Stefan Wabnitz ${ }^{3}$

1 Dipartimento di Fisica, Istituto Nazionale di Fisica Nucleare, Università "La Sapienza", P.le A. Moro 2, 00185 Roma, Italy.

2 Dipartimento di Elettronica per l'Automazione, Università di Brescia, Via Branze 38, 25123 Brescia, Italy.

3 Institut Carnot de Bourgogne, UMR 5209 CNRS, Université de Bourgogne, 9 Av. A. Savary BP 46870, 21078 Dijon, France

\begin{abstract}
Boomerons are described as accelerated solitons for special integrable systems of coupled wave equations. A general formalism based on the Lax pair method is set up to introduce such systems which look of Nonlinear Schrödingertype with linear, quadratic and cubic coupling terms. The one-soliton solution of such general systems is also briefly discussed. We display special instances of wave systems which are of potential interest for applications, including dispersion-less models of resonating waves. Among these, special attention and details are given to the celebrated equations describing the resonant interaction of three waves, in view of their application to optical pulse propagation in quadratic nonlinear media. For this particular case, we present exact solutions of the three-wave resonant interaction system, in the form of triplets moving with a common nonlinear velocity (simultons). The simultons have nontrivial phase-fronts and exist for different velocities and energy flows. We studied simulton stability upon propagation, and found that solitons with a velocity greater than a certain critical value are stable. We explore a novel consequence of the particle-like nature of three-wave simultons, namely their inelastic scattering with particular linear waves. Such phenomenon is associated with the excitation (decay) of stable (unstable) simultons by means of the absorption (emission) of the energy carried by a particular isolated pulse. Inelastic processes are exactly described in terms of boomerons. We also briefly consider collisions between different three-wave simultons.
\end{abstract}

\section{Introduction}

The propagation of nonlinear waves is a broad research subject, which is of long-standing interest to a broad community of researchers, ranging from applied mathematics to phenomenology in various physical contexts. Mathematical modeling of nonlinear wave propagation generally requires the introduction of nonlinear partial differential equations, whose investigation is a very difficult subject in itself. An epochal step in our understanding of nonlinear waves occurred with the discovery that some of such nonlinear models turn out to be integrable. When looking back about sixty years in history, we may recognize that the beginning of modern soliton theory, namely the discovery by Gardner, Green, Kruskal and Miura [1] of the method of solution of the Korteweg-de Vries (KdV) equation, was triggered by a nonlinear lattice wave

\footnotetext{
a e-mail: antonio.degasperis@roma1.infn.it
} 
problem (the celebrated Fermi-Pasta-Ulam problem [2]). Indeed, besides all complicacies of the spectral methods and algebraic structures which form the hard body of the theory, the soliton solutions remain, for very good reasons, the best known and most important character of the story. In short, soliton solutions are solitary non-dispersive waves which, for scalar wave equations, generally look as bell-shaped profiles which may be simply characterized by few parameters such as their amplitude, width and velocity. Popular examples in $1+1$ dimensions are the soliton solution

$$
u(x, t)=\frac{A}{\cosh ^{2}[p(x-v t)]}
$$

of the KdV equation

$$
u_{t}+c_{1} u_{x}+c_{3}\left(u_{x x x}+6 u u_{x}\right)=0,
$$

as well as the envelope soliton solution

$$
u(x, t)=\frac{A e^{i(k x-\omega t)}}{\cosh [p(x-v t)]}
$$

of the nonlinear Schrödinger (NLS) equation

$$
u_{t}=i c_{0} u+c_{1} u_{x}+i c_{2}\left(u_{x x}+2|u|^{2} u\right) .
$$

In these two examples, the pulse amplitude $A$, width $1 / p$ and velocity $v$ are constant parameters. Besides, the mutual relations among these parameters, namely $A=2 p^{2}, v=c_{1}+4 c_{3} p^{2}$ for the $\mathrm{KdV}$ equation, and $A=p \exp (i \theta), v=-c_{1}+2 c_{2} k$ (together with $\left.\omega=-c_{0}+c_{2}\left(k^{2}-p^{2}\right)\right)$ for the NLS equation, are distinctive of each corresponding nonlinear wave model, and provide a basic prediction for the observational data. Indeed these solitary waves are really simple objects, in that they behave as free particles. Moreover, the integrability character of the nonlinear wave model equations only comes into play in the process of collision between different solitons. A natural observation which can be made, is that the above discussed soliton behavior is so simple because the field variable is just a scalar. Therefore, in the search for solitons with non trivial motion properties, one may look for multi-component fields, with the expectation that the coupling between the different wave components could produce a far richer soliton phenomenology. To this end, generalizations of known integrable models were introduced rather soon in soliton theory, by letting the field variable $u(x, t)$ take values in a vector space or in a matrix algebra. However, in these first attempts the soliton solutions were, once again, relatively simple solitary waves. For instance, the matrix version [3] of the $\operatorname{KdV}$ equation (2) (here $\{A, B\}$ is the anticommutator $A B+B A$ )

$$
U_{t}+c_{1} U_{x}+c_{3}\left(U_{x x x}+3\left\{U, U_{x}\right\}\right)=0,
$$

$U$ being a $N \times N$ matrix, has the soliton solution

$$
U(x, t)=\frac{A}{\cosh ^{2}[p(x-v t)]} P
$$

where $P$ is a constant projector, $P^{2}=P$, and the parameters $A, p$ and $v$ are related to each other as in the scalar case (1). Similarly, in the two component Manakov [4] generalization of the NLS equation

$$
\begin{aligned}
& u_{t}^{(1)}=i c_{0} u^{(1)}+c_{1} u_{x}^{(1)}+i c_{2}\left[u_{x x}^{(1)}+2\left(\left|u^{(1)}\right|^{2}+\left|u^{(2)}\right|^{2}\right) u^{(1)}\right] \\
& u_{t}^{(2)}=i c_{0} u^{(2)}+c_{1} u_{x}^{(2)}+i c_{2}\left[u_{x x}^{(2)}+2\left(\left|u^{(1)}\right|^{2}+\left|u^{(2)}\right|^{2}\right) u^{(2)}\right]
\end{aligned}
$$

the soliton behavior is the same as in the scalar case (3), namely

$$
\left(\begin{array}{l}
u^{(1)}(x, t) \\
u^{(2)}(x, t)
\end{array}\right)=\frac{A e^{i(k x-\omega t)}}{\cosh [p(x-v t)]}\left(\begin{array}{c}
\alpha_{1} \\
\alpha_{2}
\end{array}\right)
$$


where $\left(\alpha_{1}, \alpha_{2}\right)$ is a constant unit complex vector, $\left|\alpha_{1}\right|^{2}+\left|\alpha_{2}\right|^{2}=1$.

The decisive step was taken in 1976 by F. Calogero and one the authors (A.D.) [5], who realized that a full generalization of the scalar case requires that also the constant coefficients $c_{0}, c_{1}, c_{2}$ etc. in the evolution equation, see (2) and (4), should be matrices rather than scalars. The first instances of such generalized models were constructed in the $\mathrm{KdV}$-type hierarchy where even the lowest member, which features only first-order derivatives, is a non-trivial one. This is the case of the matrix equation

$$
U_{t}+i\left[C_{0}, U\right]+\frac{1}{2}\left\{C_{1}, U_{x}\right\}+[W, U]=0, W_{x}=\frac{1}{2}\left[C_{1}, U\right], W(-\infty, t)=0,
$$

where now the constant coefficients $C_{0}$ and $C_{1}$ (as well as the dependent variables $U$ and $W$ ) are $N \times N$ matrices. Moreover the requirement that these coefficients are matrices forces the introduction of the "auxiliary" field $W(x, t)$. The soliton solution of equation (9) takes the form

$$
U(x, t)=\frac{A}{\cosh ^{2}\{p[x-\xi(t)]\}} P(t)
$$

where the amplitude $A$ and the width $1 / p$ are the same as before, $A=2 p^{2}$, but the soliton position $\xi(t)$ is no longer a linear function of time as in the case of a free particle. This happens because the soliton speed $d \xi(t) / d t$ is related to the projection matrix $P(t)$, which projects on a one-dimensional subspace (hence it is also termed "polarization" matrix) via the equation

$$
\frac{d \xi(t)}{d t}=\operatorname{Tr}\left[C_{1} P(t)\right]
$$

while the polarization matrix $P(t)$ itself satisfies the nonlinear ordinary differential equation [6]

$$
\frac{d P(t)}{d t}=\left[p C_{1}-i C_{0}, P(t)\right]+2 p P(t) C_{1}[\mathbf{1}-P(t)]
$$

Equation (10) describes an accelerated soliton, as the acceleration $d^{2} \xi(t) / d t^{2}$ is not vanishing, and its motion is that of a particle in a non constant potential. Indeed the simplest instance of equation of the type (9) is provided by the so-called "Boomeron equation" [7], which in the $2 \times 2$ case yields the following system describing the coupling of a scalar field $z(x, t)$ with two 3 -dimensional vector fields $\mathbf{u}(x, t)$ and $\mathbf{w}(x, t)$

$$
z_{t}=\mathbf{b} \cdot \mathbf{u}_{x}, \mathbf{u}_{t}=\mathbf{a} \wedge \mathbf{u}+z_{x} \mathbf{b}+\mathbf{w} \wedge \mathbf{u}, \mathbf{w}_{x}=-2 \mathbf{b} \wedge \mathbf{u}
$$

whose soliton solution reads $z=A / \cosh ^{2}\{p[x-\xi(t)]\}, \mathbf{u}=z \hat{\mathbf{n}}(t), \mathbf{w}=-2 p\left(1+\tanh ^{2}\{p[x-\right.$ $\xi(t)]\}) \mathbf{b} \wedge \hat{n}(t)$. Here $\mathbf{a}$ and $\mathbf{b}$ are arbitrary constant 3 -dimensional vectors, and the unit $3-$ dimensional vector $\hat{\mathbf{n}}(t)$ is the polarization of the soliton whose time-dependence is given by the equation

$$
\frac{d \hat{\mathbf{n}}}{d t}=\mathbf{a} \wedge \hat{\mathbf{n}}-2 p(\mathbf{b} \wedge \hat{\mathbf{n}}) \wedge \hat{\mathbf{n}}, \quad \hat{\mathbf{n}} \cdot \hat{\mathbf{n}}=1,
$$

which, in the special case $\mathbf{a} \wedge \mathbf{b}=0$, coincides with the Landau-Lifshitz equation for ferromagnetic materials. The explicit solution of the evolution equation (14) for the polarization vector $\hat{\mathbf{n}}(t)$, together with the expression

$$
\frac{d \xi(t)}{d t}=-\mathbf{b} \cdot \hat{\mathbf{n}}(t)
$$

of the soliton velocity, shows that the soliton moves as a particle in a potential barrier in such a way that its velocity at very large times becomes constant (as for a free particle) but with different velocities in the past $(t=-\infty)$ and in the future $(t=+\infty)$. This implies that, in appropriate systems of reference, the soliton in the future goes back to the same region where it came from in the past, like a boomerang. This peculiar behavior motivates the introduction 
of the term "boomeron" for describing such a soliton. Moreover, and less generically, by appropriately choosing the soliton parameters and the coefficient vectors $\mathbf{a}$ and $\mathbf{b}$, the soliton motion may even coincide with that of a particle trapped in a binding potential, with the end result that the soliton position oscillates with periodic motion in a confined space interval. In this particular case, the soliton has been termed "trappon". These findings were initially considered as a mere curiosity, because of the lack of any apparent connection to a physically observable model or application. Quite recently [8], a reduction of the boomeron equation (13), the so called "zoomeron" (or Calapso) equation, was found to be related to isothermal surfaces, and indeed it was already introduced almost a century ago in differential geometry by the italian geometer Pasquale Calapso. In the last few years, different integrable wave equations of boomeronic type were constructed and applied to systems of coupled equations, in particular of NLS-type [9, 10]. Moreover, the search of models of physical and applicative interest has progressed until several of such models have been found. One such example is the following model (which may be considered as an extension of the Manakov system, see(7)):

$$
\begin{aligned}
& u_{t}^{(1)}=i c_{0} u^{(2)}+c_{1} u_{x}^{(1)}+w u^{(2)}+i c_{2}\left[u_{x x}^{(1)}+2\left(\left|u^{(1)}\right|^{2}+\left|u^{(2)}\right|^{2}\right) u^{(1)}\right] \\
& u_{t}^{(2)}=i c_{0} u^{(1)}-c_{1} u_{x}^{(2)}-w^{*} u^{(1)}+i c_{2}\left[u_{x x}^{(2)}+2\left(\left|u^{(1)}\right|^{2}+\left|u^{(2)}\right|^{2}\right) u^{(2)}\right] \\
& w_{x}=2 c_{1} u^{(1)} u^{(2) *}, w(-\infty, t)=0
\end{aligned}
$$

whose soliton solution (compare with (8))

$$
\left(\begin{array}{l}
u^{(1)}(x, t) \\
u^{(2)}(x, t)
\end{array}\right)=\frac{A e^{i(k x-\omega t)}}{\cosh \{p[x-\xi(t)]\}}\left(\begin{array}{c}
\alpha_{1}(t) \\
\alpha_{2}(t)
\end{array}\right),\left|\alpha_{1}(t)\right|^{2}+\left|\alpha_{2}(t)\right|^{2}=1,
$$

does indeed feature a boomeronic behavior, as implied by the evolution equation of its 2dimensional complex unit polarization vector, namely $\hat{\alpha}(t)=\left(\alpha_{1}(t), \alpha_{2}(t)\right)$,

$$
\frac{d \alpha_{1}}{d t}=i c_{0} \alpha_{2}+2 p c_{1}\left|\alpha_{2}\right|^{2} \alpha_{1}, \frac{d \alpha_{2}}{d t}=i c_{0} \alpha_{1}+2 p c_{1}\left|\alpha_{1}\right|^{2} \alpha_{2}
$$

as well as the evolution equation of the soliton center

$$
\frac{d \xi(t)}{d t}=c_{1}\left(\left|\alpha_{2}(t)\right|^{2}-\left|\alpha_{1}(t)\right|^{2}\right)+2 c_{2} k
$$

where, as before, $A=p \exp (i \theta), \omega=c_{2}\left(k^{2}-p^{2}\right)$. Again here the soliton speed is time-dependent since it is related by (19) to the polarization vector $\left(\alpha_{1}(t), \alpha_{2}(t)\right)$, which satisfies the nonlinear equations (18). Alternatively, the nonlinear ODEs (18-19) may be either directly integrated (with some effort) or, more easily, the solution $\left(\alpha_{1}(t), \alpha_{2}(t)\right)$ may be obtained from the Lax pair by the Darboux-dressing technique [11,12]. Once again, the soliton (17) features both boomeronic and trapponic behaviors and, more importantly, the system of nonlinear PDEs (16) is per se of great interest in nonlinear optics applications. In fact, equations (16) model wave propagation with quadratic and cubic Kerr-type coupling, in conjunction with Schrödingertype dispersion. Quite interestingly, a reduced case of the system (16), which is obtained by letting the dispersion coefficient vanish, i.e., $c_{2}=0$, and by giving the independent variables $x$ and $t$ the exchanged role of time and, respectively, space, is the celebrated integrable model of the interaction of three resonant waves $[13,14]$. Thus we may conclude that also this well-known model exhibits soliton solutions of boomeronic type [15]. Because of the high physical relevance of the three-wave model, the main focus of this article will be the discussion of the properties of its boomeron-type soliton solutions with particular reference to nonlinear optics. In the next Section 2 we briefly review the general mathematical setting of the construction of matrix integrable wave equations based on the Lax pair approach. We shall limit ourselves to display only one model of coupled wave equations, namely the system (16), which is derived by means of a reduction from general matrix equations. Next in Section 3 we will detail the properties of a family of boomeronic solutions of the three-wave resonant interaction equations. Namely, we will discuss the stability, the asymptotic states and the collisions of three-wave boomerons. Finally, an outlook for further research and applications of accelerated solitons is presented in Section 4. 


\section{Matrix approach to integrable wave coupling}

The universal applicability of the NLS equation (4) as a model for the amplitude modulation of waves with weak dispersion and nonlinearity justifies our search for multi-component versions of such equation. The natural approach for constructing integrable multi-component NLS-type equations involves starting with the Lax pair that is associated with the scalar NLS equation (4), and replacing scalar dynamical variables with (generally rectangular) matrices [9]. In fact, we set up now this method in such a way that the scalar NLS equation (4) is recovered as just the lowest dimensional case. All systems of coupled PDEs that we may construct in this way $[9,10]$ are special (reduced) cases of the following "mother" matrix PDE:

$$
\begin{aligned}
& Q_{t}=\left[C^{(0)}, Q\right]+\sigma\left[C^{(1)}, Q_{x}\right]-\sigma\{Q, W\}-i c_{2} \sigma\left(Q_{x x}-2 Q^{3}\right) \\
& W_{x}=\left[C^{(1)}, Q^{2}\right]
\end{aligned}
$$

where the dependent variables $Q=Q(x, t)$ and $W=W(x, t)$ are $\left(N^{(+)}+N^{(-)}\right) \times\left(N^{(+)}+N^{(-)}\right)$ block matrices of the form

$$
Q=\left(\begin{array}{cc}
\mathbf{0}_{N^{(+)} \times N^{(+)}} & Q^{(+)} \\
Q^{(-)} & \mathbf{0}_{N^{(-)} \times N^{(-)}}
\end{array}\right), \quad W=\left(\begin{array}{cc}
W^{(+)} & \mathbf{0}_{N^{(+)} \times N^{(-)}} \\
\mathbf{0}_{N^{(-)} \times N^{(+)}} & W^{(-)}
\end{array}\right)
$$

In self-evident notation, the diagonal entries $W^{(+)}, W^{(-)}$are square matrices of dimension $N^{(+)} \times N^{(+)}$and $N^{(-)} \times N^{(-)}$, while the two off-diagonal rectangular blocks $Q^{(+)}$and $Q^{(-)}$, are $N^{(+)} \times N^{(-)}$or $N^{(-)} \times N^{(+)}$matrices, respectively, where $N^{(+)}$and $N^{(-)}$are arbitrary, positive integers. Here, and in the following, the constant diagonal matrix $\sigma$ in (20) is, in self-evident notation,

$$
\sigma=\left(\begin{array}{cc}
\mathbf{1}_{N^{(+)} \times N^{(+)}} & \mathbf{0}_{N^{(+)} \times N^{(-)}} \\
\mathbf{0}_{N^{(-)} \times N^{(+)}} & -\mathbf{1}_{N^{(-)} \times N^{(-)}}
\end{array}\right)
$$

while $C^{(0)}, C^{(1)}$ are arbitrary constant block-diagonal matrices,

$$
C^{(j)}=\left(\begin{array}{cc}
C^{(j)(+)} & \mathbf{0}_{N^{(+)} \times N^{(-)}} \\
\mathbf{0}_{N^{(-)} \times N^{(+)}} & C^{(j)(-)}
\end{array}\right), \quad j=0,1,
$$

and the block-diagonal matrix $W=W(x, t)$ is an auxiliary dependent variable. As usual $[A, B]$ and $\{A, B\}$ are the commutator $A B-B A$ and, respectively, the anticommutator $A B+B A$. The constant scalar coefficient $c_{2}$ is a real dispersion parameter.

The matrix equation (20) represents the compatibility condition for the Lax pair

$$
\psi_{x}=X \psi, \quad \psi_{t}=T \psi
$$

where $\psi, X$ and $T$ are $\left(N^{(+)}+N^{(-)}\right) \times\left(N^{(+)}+N^{(-)}\right)$square matrices, where $\psi=\psi(x, t, k)$ is a common solution of the two linear ordinary differential matrix equations (24), while $X=$ $X(x, t, k)$ and $T=T(x, t, k)$ depend on the coordinate $x$, the time $t$ and the complex spectral parameter $k$ according to the definitions

$$
\begin{gathered}
X(x, t, k)=i k \sigma+Q(x, t) \\
T(x, t, k)=2 c_{2} k[i k \sigma+Q(x, t)]+2 i k C^{(1)}+i c_{2} \sigma\left[Q^{2}(x, t)-Q_{x}(x, t)\right]-\sigma W+ \\
+\sigma\left[C^{(1)}, Q\right]+C^{(0)} .
\end{gathered}
$$

In order to simplify the notation, from now on (and as already done in the previous formula) we do not specify the dimension of the matrices $\mathbf{0}$ and $\mathbf{1}$, and we may even omit to write the matrix 1 altogether, as we trust the reader will not be confused by this omission.

Let us now consider of the condition

$$
Q^{\dagger}(x, t)=S Q(x, t) S
$$


on the solution $Q(x, t)$ of the matrix evolution equation (20) where the dagger stands for hermitian conjugation. The constant matrix $S$ is block diagonal,

$$
S=\left(\begin{array}{cc}
S^{(+)} & \mathbf{0} \\
\mathbf{0} & S^{(-)}
\end{array}\right)
$$

its off-diagonal blocks are vanishing rectangular matrices, while its diagonal blocks $S^{(+)}$and $S^{(-)}$are $N^{(+)} \times N^{(+)}$or $N^{(-)} \times N^{(-)}$, respectively, diagonal matrices whose diagonal elements $s_{n}^{( \pm)}$(with no loss of generality) are signs. Namely,

$$
S^{( \pm)}=\operatorname{diag}\left(s_{1}^{( \pm)}, \cdots, s_{N^{( \pm)}}^{( \pm)}\right), \quad s_{n}^{( \pm)^{2}}=1 .
$$

This of course implies the relations $S^{2}=\mathbf{1}, S^{(+)^{2}}=\mathbf{1}, S^{(-)^{2}}=\mathbf{1}$. The reduction equation $(27)$ is well motivated by the fact that it captures several interesting models of dispersive propagation of multicomponent waves in weakly nonlinear media. To this aim, it is convenient to rewrite the matrix PDEs (20) in terms of the blocks $Q^{(+)}, Q^{(-)}, W^{(+)}$and $W^{(-)}$, see $(21)$. These read

$$
\begin{gathered}
Q_{t}^{( \pm)}=C^{(0)( \pm)} Q^{( \pm)}-Q^{( \pm)} C^{(0)(\mp)} \pm\left[C^{(1)( \pm)} Q_{x}^{( \pm)}-Q_{x}^{( \pm)} C^{(1)(\mp)}\right] \\
\mp\left[W^{( \pm)} Q^{( \pm)}+Q^{( \pm)} W^{(\mp)}\right] \mp i c_{2}\left[Q_{x x}^{( \pm)}-2 Q^{( \pm)} Q^{(\mp)} Q^{( \pm)}\right] \\
W_{x}^{( \pm)}=\left[C^{(1)( \pm)}, Q^{( \pm)} Q^{(\mp)}\right] .
\end{gathered}
$$

where $C^{(j)(+)}$ and $C^{(j)(-)}$, are the $N^{(+)} \times N^{(+)}$, or $N^{(-)} \times N^{(-)}$, respectively, constant square matrix blocks of $C^{(j)}$, see $(23)$. The reduction condition $(27)$ is accounted for by introducing the dependent variable $U(x, t)$ through the definitions

$$
Q^{(-)}(x, t)=U(x, t), \quad Q^{(+)}(x, t)=S^{(+)} U^{\dagger}(x, t) S^{(-)} .
$$

Since the constant $c_{2}$ is real, the above expressions of $Q^{(+)}$and $Q^{(-)}$in terms of the single variable $U(x, t)$ are compatible with the equations $(30)$, which then reduce to

$$
\begin{gathered}
U_{t}=C^{(0)(-)} U-U C^{(0)(+)}-\left[C^{(1)(-)} U_{x}-U_{x} C^{(1)(+)}\right]+ \\
+\left[W^{(-)} U+U W^{(+)}\right]+i c_{2}\left[U_{x x}-2 U S^{(+)} U^{\dagger} S^{(-)} U\right] \\
W_{x}^{(+)}=\left[C^{(1)(+)}, S^{(+)} U^{\dagger} S^{(-)} U\right] \\
W_{x}^{(-)}=\left[C^{(1)(-)}, U S^{(+)} U^{\dagger} S^{(-)}\right] .
\end{gathered}
$$

Here $U$ is an $N^{(-)} \times N^{(+)}$rectangular matrix, (see (32) and (21)), while the variables $W^{( \pm)}(x, t)$ are square matrices: $W^{(+)}$is an $N^{(+)} \times N^{(+)}$matrix and $W^{(-)}$is an $N^{(-)} \times N^{(-)}$matrix, respectively, and it is easily seen that they satisfy the "hermitianity" conditions

$$
W^{(+)}=-S^{(+)} W^{(+) \dagger} S^{(+)}, \quad W^{(-)}=-S^{(-)} W^{(-) \dagger} S^{(-)} .
$$

Similarly, the constant matrices $C^{(j)( \pm)}$ satisfy the following conditions:

$$
C^{(j)( \pm)}=-(-1)^{j} S^{( \pm)} C^{(j)( \pm) \dagger} S^{( \pm)}, \quad j=0,1
$$

As for the "sign" matrices $S^{(+)}$and $S^{(-)}$, see (29), we note that one could set, for instance, $s_{1}^{(+)}=1$ with no loss of generality, but we prefer to keep the symmetrical, though redundant, notation (29).

We observe that the general matrix nonlinear evolution equation (33) may be well specialized to quite a large family of coupled-wave equations by playing with various choices of the integers $N^{(+)}$and $N^{(-)}$, and of the constant matrix coefficients $C^{(j)(+)}$ and $C^{(j)(-)}$. This exercise is 
certainly worth doing since it happens that among this family of wave equations there are some models which look interesting in applicative contexts. While we refer the reader to [9] and [10] for the derivation of other model wave equations, we specify here the values of the integers $N^{(+)}, N^{(-)}$and the matrix coefficients $C^{(j)(+)}$ and $C^{(j)(-)}$ which correspond to the system (16). This system of two coupled NLS equations is obtained for $N^{(+)}=1$ and $N^{(-)}=2$, together with $S^{(+)}=1$ and $S^{(-)}=\operatorname{diag}(-1,-1)$. Here the choice of the matrix coefficients is

$$
C^{(0)(+)}=C^{(1)(+)}=0, \quad C^{(0)(-)}=\left(\begin{array}{cc}
0 & i c_{0} \\
-i c_{0} & 0
\end{array}\right), \quad C^{(1)(-)}=\left(\begin{array}{cc}
-c_{1} & 0 \\
0 & c_{1}
\end{array}\right)
$$

while the dependent variables are the two components $\left(u^{(1)}(x, t), u^{(2)}(x, t)\right)$ of the 2-dimensional (column) vector $U$ and the function $w(x, t)$ is defined through the off-diagonal matrix $W^{(-)}$

$$
W^{(-)}=\left(\begin{array}{cc}
0 & w \\
-w^{*} & 0
\end{array}\right)
$$

while $W^{(+)}=0$. The case in which the dispersion coefficient vanishes, $c_{2}=0$, coincides with the model of resonant interaction of three waves. The boomeron solutions of this system can be obtained by applying the Darboux-dressing method [11] to the Lax pair (24): the rich phenomenology of these accelerated solitons is the content of the next section.

\section{Propagation, stability and scattering of parametric three-wave solitons}

Three-wave resonant interaction (TWRI) is ubiquitous in various branches of science, as it describes the mixing of waves with different frequencies in weakly nonlinear and dispersive media. The TWRI model is typically encountered in the description of any conservative nonlinear medium where the nonlinear dynamics can be considered as a perturbation of the linear wave solution, the lowest-order nonlinearity is quadratic in the field amplitudes and the phasematching (or resonance) condition is satisfied. After the first milestone studies on TWRI [13, 16-22], many theoretical investigations were made in the context of nonlinear optics, plasma physics, acoustics, fluid dynamics, and solid state physics. TWRI has been extensively studied in the context of nonlinear optics, since it applies to parametric amplification, second harmonic generation and frequency conversion, stimulated Raman and Brillouin scattering and light speed control [23-27]. TWRI also occurs in plasmas in the saturation of parametric decay instabilities, radio frequency heating and laser-plasma interactions $[17,18,28-30]$. In the TWRI scenarios, parametric TWRI solitons play a pivotal role because of their particle-like behavior which makes them important mechanisms of energy transport, waves manipulation and processing. The particle-like behavior of solitons is responsible for numerous phenomena, which cannot occur with linear waves. Two classes of analytical soliton solutions of the TWRI have been known for over three decades. The first type of solitons describes the mixing of three pulses which travel with their respective linear group velocity, and interact for just a short time [13,20-22,31]. The second type of solitons, also known as simultons, are formed as a result of the mutual trapping of pulse envelopes at the three different frequencies. Hence the three wave packets travel locked together with a common group velocity [16-19]. In this section we present a new three-parameter family of bright-bright-dark simultons that travel with a common, locked velocity [32]. The most remarkable physical property of the present simultons is that their speed can be continuously varied by means of adjusting the energy of the two bright pulses. We have investigated the propagation stability of TWRI simultons (TWRIS) and we have found that a stable triplet loses its stability as soon as its velocity decreases below a well defined critical value. Another feature of a TWRIS is that an unstable triplet decays into a stable one through the emission of a pulse, followed by acceleration up to reaching a constant velocity. Moreover, a stable triplet may be excited into an unstable simulton by slowing down as a result of the absorption of an isolated wave [33]. Such processes are exactly described in terms of an analytical higher-order soliton solution with varying speed, or boomeron. The 
present TWRIS scattering process may be pictured as the interaction of radiation with a twolevel atomic system: transitions among excited and ground soliton states are induced by the absorption and spontaneous emission of a wave. To conclude our investigations on TWRI solitons we have also performed numerical studies on TWRIS collisions, finding that TWRIS with different speeds are stable upon collision, and TWRIS with equal speeds interact with each other in a way which is strongly dependent upon their initial relative phase.

\subsection{Three-wave interaction equations}

For our convenience, let us first rewrite the coupled partial differential equations (PDEs) (16) with vanishing dispersion, $c_{2}=0$, as [13]:

$$
\begin{aligned}
& E_{1 t}-V_{1} E_{1 z}=E_{2}^{*} E_{3}^{*}, \\
& E_{2 t}-V_{2} E_{2 z}=-E_{1}^{*} E_{3}^{*}, \\
& E_{3 t}-V_{3} E_{3 z}=E_{1}^{*} E_{2}^{*},
\end{aligned}
$$

where the subscripts $t$ and $z$ denote derivatives in the longitudinal and transverse dimension, respectively. Moreover, $E_{n}=E_{n}(z, t)$ are the complex amplitudes of the three waves, $V_{n}$ are their linear velocities, and $n=1,2,3$. For the reader's benefit, we provide here the transformation of the dependent and independent variables which maps the system (16) into the form (40). The coordinates $x$ and $t$ in (16) are related to the new coordinates $z$ and $t^{\prime}$ by the formula

$$
\begin{aligned}
z & =\frac{1}{2}\left(V_{1} V_{3}+V_{2} V_{3}-2 V_{1} V_{2}\right) t+\frac{1}{2 c_{1}} V_{3}\left(V_{2}-V_{1}\right) x, \\
t^{\prime} & =\frac{1}{2 c_{1}}\left(V_{1}-V_{2}\right) x+\frac{1}{2}\left(V_{1}+V_{2}-2 V_{3}\right) t,
\end{aligned}
$$

while the relation between amplitudes reads

$$
\begin{aligned}
E_{1} & =\sqrt{\frac{V_{2}-V_{3}}{\rho}} u^{(1) *}, E_{2}=\sqrt{\frac{V_{1}-V_{3}}{\rho}} u^{(2)}, E_{3}=\sqrt{\frac{V_{1}-V_{2}}{4 c_{1}^{2} \rho}}\left(w+i c_{0}\right), \\
\rho & =\left(V_{1}-V_{2}\right)\left(V_{1}-V_{3}\right)\left(V_{2}-V_{3}\right) /\left(4 c_{1}^{2}\right) .
\end{aligned}
$$

Moreover, for notational convenience, we replace in (40) the new time variable $t^{\prime}$ with $t$. We assume here the ordering $V_{1}>V_{2}>V_{3}$ which, together with the above choice of signs before the quadratic terms, entails the non-explosive character of the interaction. In the following, with no loss of generality, we shall write the equations (40) in a reference frame such that $V_{3}=0$. A remarkable property of the equations (40) is their invariance with respect to the transformation

$$
\hat{E}_{n}(z, t)=s \exp \left[i\left(q_{n} z_{n}+\alpha_{n}\right)\right] E_{n}\left(s z+z_{0}, s t+t_{0}\right)
$$

where $\alpha_{1}+\alpha_{2}+\alpha_{3}=0, q_{n}=q\left(V_{n+1}-V_{n+2}\right), z_{n}=z+V_{n} t$ are the characteristic coordinates and $n=1,2,3 \bmod (3)$. As the transformation (43) depends on six real parameters, namely $\alpha_{1}, \alpha_{2}, s, q, z_{0}$ and $t_{0}$, clearly one may introduce these parameters in the expression of any given solution of the TWRI equation. The evolution equations (40) represent an infinite-dimensional Hamiltonian dynamical system, with the conserved Hamiltonian

$$
\begin{aligned}
& H=\frac{1}{4 i} \int_{-\infty}^{+\infty}\left[V_{1}\left(E_{1 z}^{*} E_{1}-E_{1 z} E_{1}^{*}\right)-V_{2}\left(E_{2 z}^{*} E_{2}-E_{2 z} E_{2}^{*}\right)+\right. \\
& \left.+V_{3}\left(E_{3 z}^{*} E_{3}-E_{3 z} E_{3}^{*}\right)+2 E_{1} E_{2} E_{3}-2 E_{1}^{*} E_{2}^{*} E_{3}^{*}\right] d z,
\end{aligned}
$$

energies (Manley-Rowe invariants)

$$
I_{12}=I_{1}+I_{2}=\frac{1}{2} \int_{-\infty}^{+\infty}\left(\left|E_{1}\right|^{2}+\left|E_{2}\right|^{2}\right) d z
$$




$$
I_{23}=I_{2}+I_{3}=\frac{1}{2} \int_{-\infty}^{+\infty}\left(\left|E_{2}\right|^{2}+\left|E_{3}\right|^{2}\right) d z
$$

and total transverse momentum

$$
J=\frac{1}{4 i} \int_{-\infty}^{+\infty}\left[\left(E_{1}^{*} E_{1 z}-E_{1} E_{1 z}^{*}\right)-\left(E_{2}^{*} E_{2 z}-E_{2} E_{2 z}^{*}\right)+\left(E_{3}^{*} E_{3 z}-E_{3} E_{3 z}^{*}\right)\right] d z .
$$

Each of the above conserved quantities (45)-(47) is related to a given internal parameter of the TWRIS which, in turn, is associated with a symmetry (e.g., phase rotation or space translation) of the TWRI equations (40) [34]. As a consequence, one may expect that equation (40) possess a three-parameter family of soliton solutions.

\subsection{Three-parameters simulton family}

In this section, we will discuss the recently found simulton family of solutions of equations (40) [32], by using the general results on TWRI equations which were presented in Ref. [15]. The expression of these simultons is

$$
\begin{gathered}
E_{1}=\frac{2 p a}{\sqrt{|b|^{2}+a^{2}}} \frac{g_{1}}{g\left(V_{1}-V_{2}\right)} \frac{\exp \left[i\left(q_{1} z_{1}-\chi z+\omega t\right)\right]}{\cosh [B(z+V t)]}, \\
E_{2}=\frac{-2 p b}{\sqrt{|b|^{2}+a^{2}}} \frac{g_{2}}{g\left(V_{1}-V_{2}\right)} \frac{\exp \left[i\left(q_{2} z_{2}+\chi z-\omega t\right)\right]}{\cosh [B(z+V t)]}, \\
E_{3}=\left\{1+\frac{2 p b^{*}}{|b|^{2}+a^{2}}[1-\tanh [B(z+V t)]]\right\} \frac{a g_{3} \exp \left(i q_{3} z_{3}\right)}{g\left(V_{1}-V_{2}\right)}
\end{gathered}
$$

where

$$
\begin{aligned}
b & =(Q-1)(p+i k / Q), \quad r=p^{2}-k^{2}-a^{2}, \\
Q & =\frac{1}{p} \sqrt{\frac{1}{2}\left[r+\sqrt{r^{2}+4 k^{2} p^{2}}\right],} \\
B & =p\left[V_{1}+V_{2}-Q\left(V_{1}-V_{2}\right)\right] /\left(V_{1}-V_{2}\right), \\
V & =2 V_{1} V_{2} /\left[V_{1}+V_{2}-Q\left(V_{1}-V_{2}\right)\right], \\
\chi & =k\left[V_{1}+V_{2}-\left(V_{1}-V_{2}\right) / Q\right] /\left(V_{1}-V_{2}\right), \\
\omega & =-2 k V_{1} V_{2} /\left(V_{1}-V_{2}\right), \quad q_{n}=q\left(V_{n+1}-V_{n+2}\right), \\
g_{n} & =\left|\left(V_{n}-V_{n+1}\right)\left(V_{n}-V_{n+2}\right)\right|^{-1 / 2}, g=g_{1} g_{2} g_{3},
\end{aligned}
$$

and $n=1,2,3 \bmod (3)$. The above expressions depend on the six real parameters $V_{1}, V_{2}, p, k$, $q, a>0$. From the definition of $Q$, one can see that the above parameters must be chosen so that if $k=0$, then $p^{2}>a^{2}$. The TWRIS is composed of two bright pulses $(48,49)$, and a kink or shock-like pulse (50), which travel with a common locked velocity $V$ which does not coincide with any of the three characteristic velocities $V_{1}, V_{2}, V_{3}$. The expressions (48)-(50) may be represented in a more convenient form as

$$
E_{n}(\xi, \tau)=U_{n}(\xi) \exp \left[i \Phi_{n}(\xi, \tau)\right], n=1,2,3 .
$$

Here we use a reference frame which moves along with the soliton, with coordinates $\xi=z+$ $V t, \tau=t$ where $U$ and $\Phi$ are real functions and $\Phi_{n}(\xi, \tau)=\phi_{n} \tau+f_{n}(\xi)$. A simple analysis of (52) shows that, for any value of the parameters, the pulse amplitudes $U_{1}(\xi), U_{2}(\xi)$ and $U_{3}(\xi)$ are even functions of $\xi$ and the phase constants satisfy $\phi_{1}+\phi_{2}+\phi_{3}=0$. On the other hand, if $k=0$, the phase profiles are all piecewise linear in $\xi$, and obey the condition $f_{1}(\xi)+f_{2}(\xi)+f_{3}(\xi)=0$ 

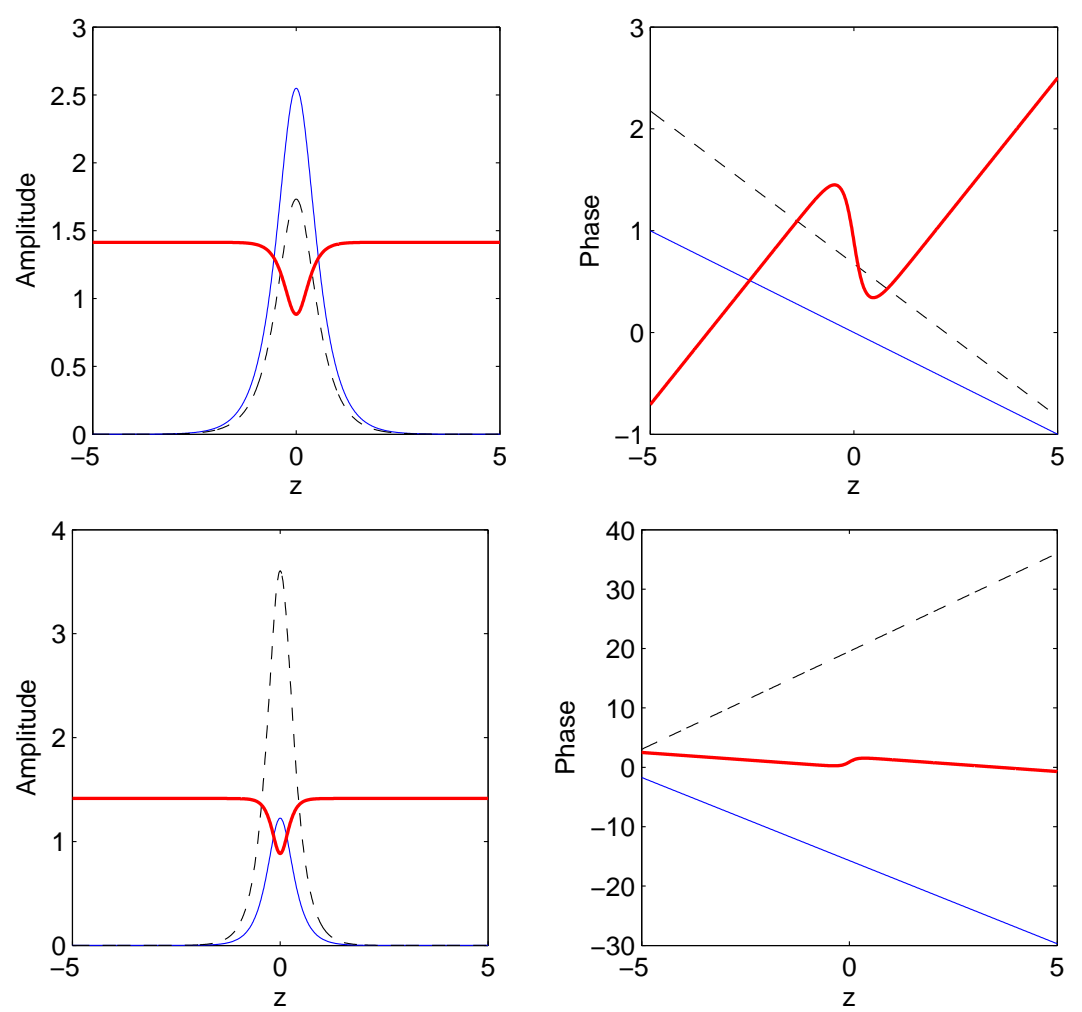

Fig. 1. Amplitude and phase of two simultons (48)-(50) at $t=0$ with $V_{1}=2, V_{2}=1, a=1, k=0.5$, $q=0.5$; in the upper figures $p=1$, in the lower figures $p=-1$. Thin solid curve: $E_{1}$; dashed curve: $E_{2} ;$ thick solid curve: $E_{3}$.

for $p \xi>0$ and $f_{1}(\xi)+f_{2}(\xi)+f_{3}(\xi)=\pi$ for $p \xi<0$. Whereas for $k \neq 0$ the phase profile $f_{3}(\xi)$ is nonlinear and $\cos \left[f_{1}(\xi)+f_{2}(\xi)+f_{3}(\xi)\right]$ is an odd function of $\xi$; moreover the kink pulse $E_{3}$ is "grey" if $k \neq 0$ and is "dark" if $k=0$. Such amplitude and phase front profiles prevent a net energy exchange among the three waves. It is important to point out that the condition $-1<Q<1$ leads to a speed $V$ that lies in-between the characteristic velocities $V_{1}$ and $V_{2}$ of the two bright pulses, i.e. $V_{1}>V>V_{2}$. The above described properties mean that TWRIS represent a significant generalization with respect to previously known three-wave solitons which exhibit a simple (constant) phase profile and correspond to the special case $k=q=0, r>0$ [16-18]. In Fig. 1 we plot two examples of TWRIS amplitude and phase-fronts (48)-(50). It is interesting to consider the physical meaning of the various TWRIS parameters appearing in (48)-(50). For a given choice of the characteristic linear velocities $V_{1}$ and $V_{2}$, we are left with the four independent parameters $p, k, q$, and $a$. We may note that $p$ is basically associated with the scaling of the wave amplitudes, as well as of the coordinates $z$ and $t$. The parameter $a$ determines the amplitude of the asymptotic plateau of the kink $E_{3}$. The value of $k$ is related to the wave-number of the soliton. The parameter $q$ adds a phase contribution which is linear in $z$ and $t$. Since the system (40) is invariant under a transformation (43), without loss of generality we may set $a=1$, which reduces the number of essential parameters to just three, corresponding to the three symmetries of equations (40). The parameters $p, k, q$ in (48)-(50) may be more conveniently mapped into the parameters $V, \phi_{1}, \phi_{2}$ of equation (52), which provide a more direct physical insight into the features of a TWRIS. Such a mapping is obtained by comparing equations (48)-(50) with (52), and reads as:

$$
V=\frac{2 V_{1} V_{2}}{V_{1}+V_{2}-Q\left(V_{1}-V_{2}\right)}
$$




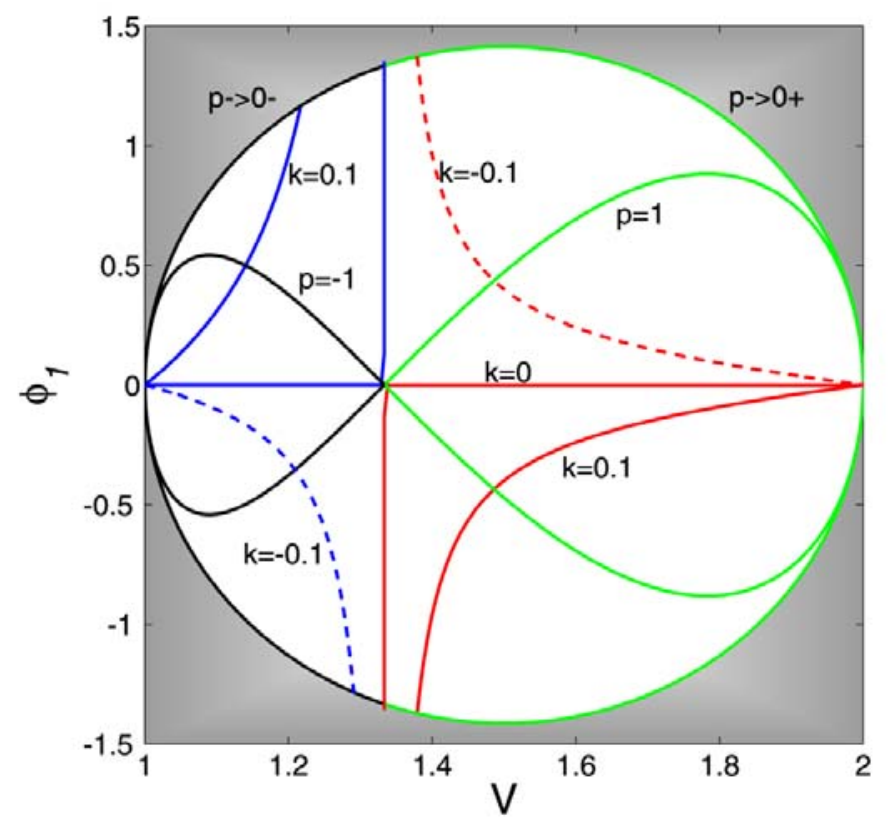

Fig. 2. Mapping of parameters $\left(V, \phi_{1}\right)$, in the space $\phi_{1}=-\phi_{2}$, into parameters $(p, k)(q=0, a=1)$, for $V_{1}=2, V_{2}=1$. Shaded areas represent forbidden parameter regions.

$$
\begin{aligned}
& \phi_{1}=q V_{2}\left(V_{1}-V\right)+\chi V+\omega, \\
& \phi_{2}=q V_{1}\left(V-V_{2}\right)-\chi V-\omega,
\end{aligned}
$$

whereas the inverse mapping:

$$
\begin{aligned}
q & =\frac{\phi_{1}+\phi_{2}}{V\left(V_{1}-V_{2}\right)} \\
k & =\frac{\phi_{1}-q V_{2}\left(V_{1}-V\right)}{V \frac{V_{1}+V_{2}}{V_{1}-V_{2}}-V / Q-\frac{2 V_{1} V_{2}}{V_{1}-V_{2}}} \\
p & =\operatorname{sign}(Q) \sqrt{\frac{Q^{2}\left(k^{2}+|a|^{2}\right)-k^{2}}{Q^{2}\left(1-Q^{2}\right)}} .
\end{aligned}
$$

In Fig. 2 we plot the mapping of the parameters $\left(V, \phi_{1}\right)$, in the subspace $\phi_{1}=-\phi_{2}$, into the new parameters $(p, k)(q=0, a=1)$. It is worth noting that the condition $k^{2}>Q^{2} a^{2} /\left(1-Q^{2}\right)$ must be satisfied in order to obtain real values of $p$. This implies that, for a fixed $V$, there exists a circular boundary for the allowed domain of phase constants $\phi_{1,2}$ (i.e., the region inside the shaded areas in Fig. 2). Therefore a TWRIS may be simply expressed in terms of its velocity $V$ and the two phase constants $\phi_{1}$ and $\phi_{2}$. Let us investigate what are the TWRIS properties for a fixed choice of the linear velocities $V_{1}$ and $V_{2}$, upon variations of its energy flows and transverse momentum. As an example, Fig. 3(a) shows the dependence of the phase constant $\phi_{1}$ on the locked velocity $V$, for the case where $\phi_{2}=-\phi_{1}$, with different values of the conserved energy $I_{12}$. Fig. 3(b) illustrates the dependence of the energies $I_{1}$ and $I_{2}$ (which happen to be timeindependent for a TWRIS) on the locked velocity $V$, for different choices of the phase constant $\phi_{1}=-\phi_{2}$. Figs. 3(c)-(d) show the dependence of the energies $I_{12}$ and of the renormalized transverse momentum $\bar{J}$ [as defined by (57), see discussion below] upon the locked velocity $V$ respectively, for different choices of the phase constant $\phi_{1}=-\phi_{2}$. As it can be seen from the 

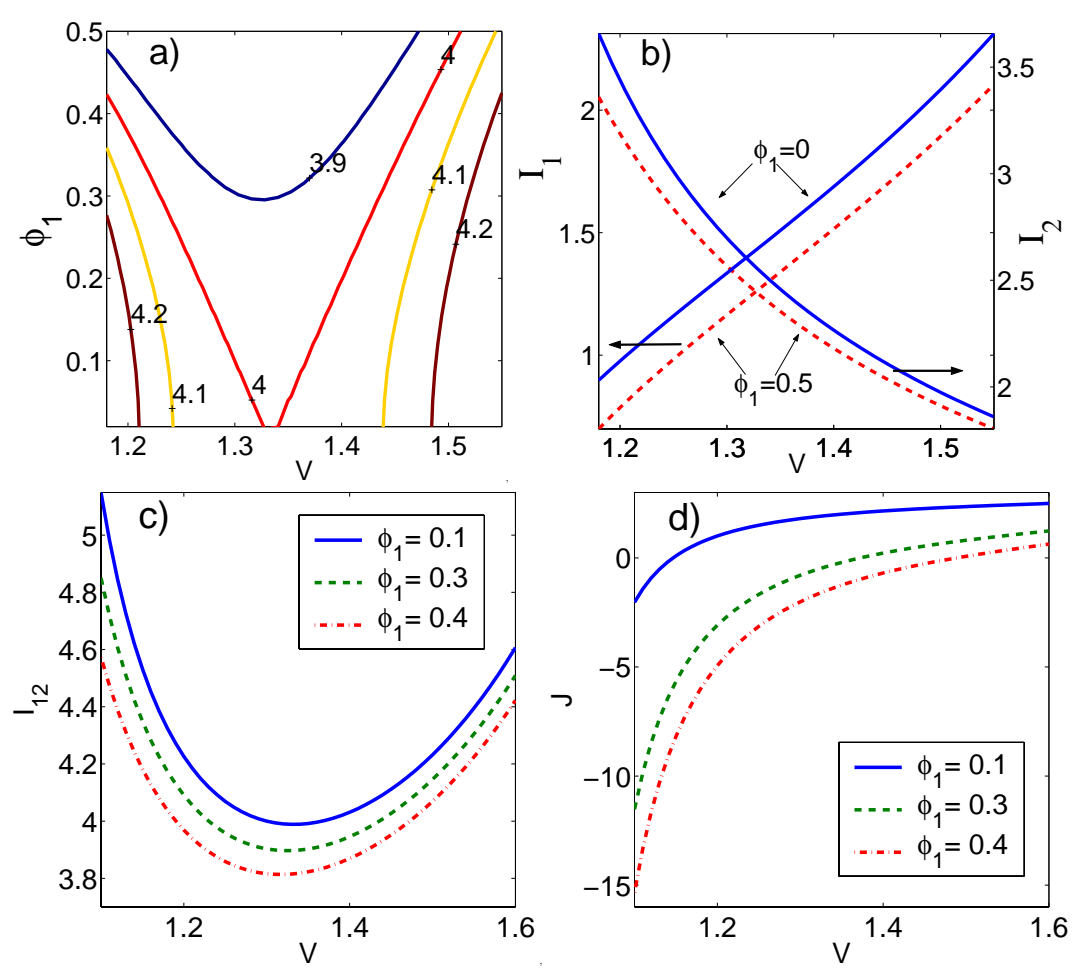

Fig. 3. (a) phase constant $\phi_{1}$ versus velocity $V$ for different energies $I_{12}$. (b) energies $I_{1}, I_{2}$ versus velocity $V$ for representative values of the phase constant $\phi_{1}$. (c) energies $I_{12}$ versus velocity $V$ for representative values of the phase constant $\phi_{1}$. (d) transverse momentum $\bar{J}$ versus velocity $V$. In all cases $\phi_{2}=-\phi_{1}, V_{1}=2, V_{2}=1$.

above figures, the intensity, the phase profiles, as well as the energy distribution among the different wave packets strongly depend upon the value of the locked velocity $V$.

\subsection{Three-wave simulton stability}

The next crucial issue is the propagation stability of TWRIS. A first insight into this problem may be provided by performing a linear stability analysis as it was done in Ref. [35]. Let us consider a perturbed TWRIS of the form

$$
\tilde{E}_{n}(\xi, \tau)=\left(x_{n}(\xi)+P_{n}(\xi, \tau)\right) e^{i \phi_{n} \tau}, \quad n=1,2,3
$$

where $x_{n}(\xi)=U_{n}(\xi) \exp \left[i f_{n}(\xi)\right]$ is the soliton profile; for a weak perturbation, we take $\left|P_{n}\right|<<$ $\left|x_{n}\right|$. By inserting the above ansatz in equations (40), and by retaining only linear terms in $P_{n}$, one obtains a linear system of PDEs. For the numerical analysis, these PDEs can be reduced to a system of ordinary differential equations $d P(\tau) / d \tau=M P(\tau)$, by approximating the spatial derivatives with finite differences, where $P$ is the perturbation vector sampled on a finite grid. A sufficient condition for the instability of a stationary solution $x_{n}(\xi)$ is that the matrix $M$ has at least one eigenvalue with positive real part. Numerical computations over a wide parameter range show that eigenvalues of $M$ exist with a positive real part whenever $p<0$. On the other hand, for $p>0$ the largest real part of the eigenvalues is equal to zero, which means that the TWRIS are only neutrally stable. Note that the instability condition $p<0$ leads to the inequality $V<V_{c r}=2 V_{1} V_{2} /\left(V_{1}+V_{2}\right)$. Extensive numerical integrations of equations (40) confirm that TWRIS with $V<V_{c r}\left(V>V_{c r}\right)$ are always unstable (stable). The propagation of either stable or unstable TWRIS is illustrated in Fig. 4, which shows the general feature 

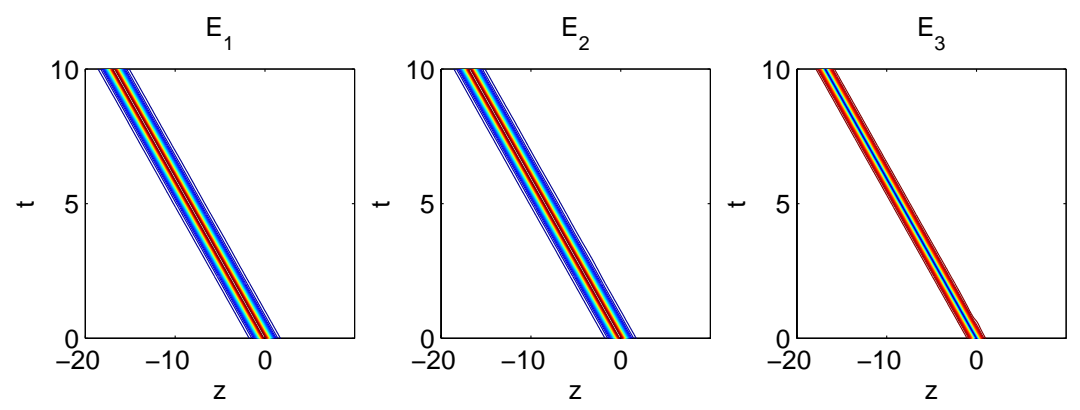

$\mathrm{E}_{1}$
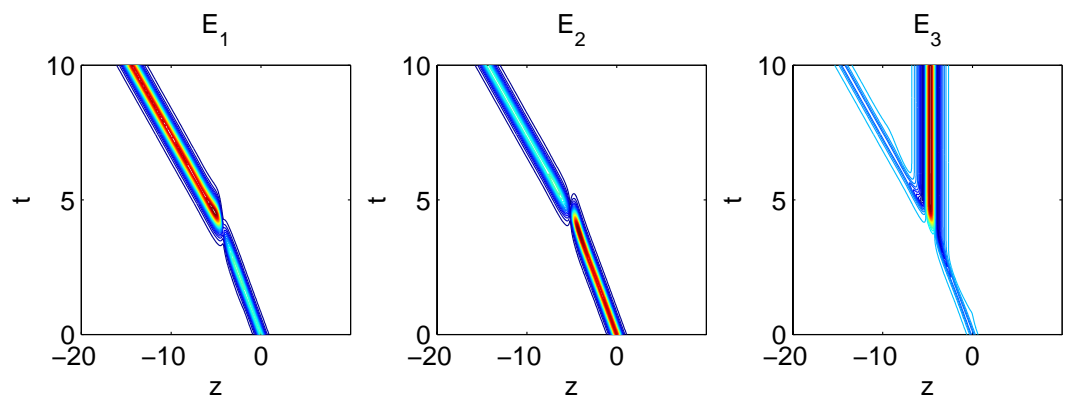

Fig. 4. Propagation of stable and unstable simultons. The common parameters are $V_{1}=2, V_{2}=1$, $a=1, k=0.5, q=1$. For the stable simulton (upper figures) $p=1\left(V=1.68>V_{c r}, \phi_{1}=0.5\right.$, $\phi_{2}=2.2$ ), whereas for the unstable simulton (lower figures) $p=-1\left(V=1.1<V_{c r}, \phi_{1}=1.43\right.$, $\left.\phi_{2}=-0.33\right)$.

of unstable solitons with $V<V_{c r}$. Namely, the simulton decays into a stable soliton with $V>V_{c r}$, and it emits a pulse in the wave $E_{3}$. It is quite remarkable that the dynamics of the decay from unstable into stable solitons may be exactly described by analytical solutions with variable velocity or boomerons, as it will be shown in the next section. We performed further investigations of TWRIS stability by carrying out a multi-scale asymptotic analysis (MAA) [36-40]. In this way, one obtains the following condition which defines the borderline between stable and unstable TWRIS

$$
G=\frac{\partial\left(I_{12}, \bar{I}_{23}, \bar{J}\right)}{\partial\left(\phi_{1}, \phi_{2}, V\right)}=0,
$$

where $G$ is the Jacobian of the constants of motion $I_{12}, \bar{I}_{23}, \bar{J}$ with respect to $\phi_{1}, \phi_{3}, V\left(\phi_{3}=\right.$ $\left.-\phi_{1}-\phi_{2}\right)$. Note that in (55), $\bar{I}_{23}$ and $\bar{J}$ are obtained by re-normalizing the divergent integrals (46) and (47) according to the prescription [41]

$$
\begin{gathered}
\bar{I}_{23}=\frac{1}{2} \int_{-\infty}^{+\infty}\left(\left|E_{2}\right|^{2}+\left|E_{3}\right|^{2}-\left|E_{30}\right|^{2}\right) d z \\
\bar{J}=J-\frac{1}{4 i} \int_{-\infty}^{+\infty}\left[\left(E_{3}^{*} E_{3 z}-E_{3} E_{3 z}^{*}\right)\left[\frac{\left|E_{30}\right|^{2}}{\left|E_{3}\right|^{2}}\right]\right] d z,
\end{gathered}
$$

where $\left|E_{30}\right|=\lim _{|z| \rightarrow \infty}\left|E_{3}\right|$ is the asymptotic amplitude of the kink . Note that the availability of exact soliton solutions allows for the analytical calculation of the above integrals, hence of the condition (55), which is an extension of the well-known Vakhitov-Kolokov criterion. The equation (55) provides a sufficient stability condition [39]. Among all of the simultons belonging to the general family (52), the criterion (55) may only be applied within the specific constraint $\phi_{1}=\phi_{2}$. Indeed, in this case we found that the condition $G=0$ leads, once again, to the previously found marginal stability condition $V=V_{c r}=2 V_{1} V_{2} /\left(V_{1}+V_{2}\right)$. Moreover, a direct insight into the global stability properties of TWRIS for all possible values of their parameters 
may be gained with a powerful geometrical approach [42]. Indeed, TWRIS may be obtained as the solutions of the variational problem

$$
\delta\left(H+\phi_{1} I_{12}-\left(\phi_{1}+\phi_{2}\right) \bar{I}_{23}-V \bar{J}\right)=0,
$$

where $\delta$ is the Frechét derivative. In other words, TWRIS represent the extrema of the Hamiltonian (44), for a fixed value of the energies and momentum (here $V, \phi_{1},-\left(\phi_{1}+\phi_{2}\right)$ represent Lagrange multipliers). Stable triplets are obtained whenever such extrema coincide with a global minimum of $H$. Clearly, if multiple solutions exist with the same $I_{12}, \bar{I}_{23}, \bar{J}$, the stable solution is obtained on the lower branch of $H$. In this framework, the condition (55) corresponds to solitons such that the normal vector to the three-dimensional surface $H=H\left(I_{12}, \bar{I}_{23}, \bar{J}\right)$ lies in the space $H=$ const. The above geometrical considerations permit the visualization of the stability boundaries when considering a projection of the hyper-surface $H=H\left(I_{12}, \bar{I}_{23}, \bar{J}\right)$ on the plane $\left(I_{12}, H\right)$. For example, Fig. 5 displays the dependence of $H$ upon $I_{12}$ for the case $\phi_{1}=-\phi_{2}$, where the criterion (55) cannot be applied, and in the case $\phi_{1}=\phi_{2}$. Here it is evident that the two branches of the Hamiltonian merge exactly at $V=V_{c r}$ : at this point, the normal to the $H$ curve is also orthogonal to the vertical axis. Interestingly enough, Fig. 5 shows that the borderline TWRIS corresponds to a minimum of the bright pulses energy $I_{12}$ with respect to $V$. To summarize, we have shown that different numerical and analytical methods concur in predicting that the TWRIS stability is determined by the condition $V>V_{c r}$.
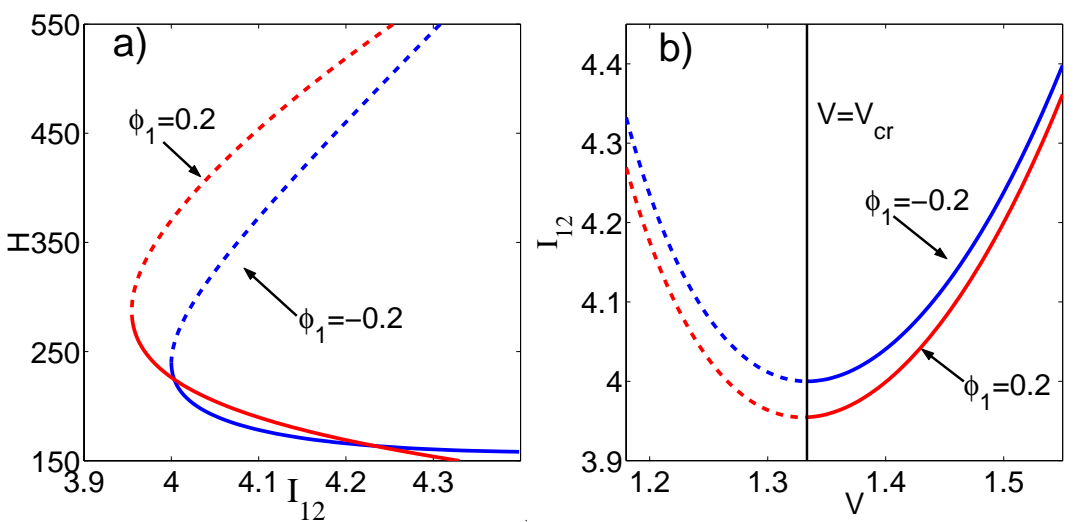

Fig. 5. (a) Hamiltonian $H$ versus energy $I_{12}$ and (b) $I_{12}$ versus velocity $V$. Characteristic velocities are $V_{1}=2, V_{2}=1$ and $\phi_{2}=-0.2$. Dashed (solid) curves correspond to unstable (stable) solitons.

\subsection{Three-wave boomeron}

The results of Fig. 4 illustrate that an unstable simulton with $V<V_{c r}$ decays into a stable simulton with $V>V_{c r}$. This process is accompanied by the emission of an isolated bright pulse in wave $E_{3}$. It is quite remarkable that the simulton decay and wave packet emission as it is numerically observed in Fig. 4 may be exactly reproduced in terms of an analytical higher-order soliton solution with varying speed, or "boomeron". Such solution was found by means of the techniques described in Ref.[15], and it can be expressed as

$$
\begin{gathered}
E_{1}=\frac{2 p V_{2}}{\Delta} \sqrt{\frac{2 V_{1}}{V_{1}-V_{2}}} e^{i q_{1} z_{1}}\left(H_{+}^{*} e^{-i \theta}-H_{-}^{*} e^{i \theta}\right), \\
E_{2}=\frac{2 p V_{1}}{\Delta} \sqrt{\frac{2 V_{2}}{V_{1}-V_{2}}} e^{i q_{2} z_{2}}\left(\sqrt{(1-Q) /(1+Q)} H_{+} e^{i(\beta+\theta)}-\sqrt{(1+Q) /(1-Q)} H_{-} e^{-i(\beta+\theta)}\right),
\end{gathered}
$$




$$
E_{3}=a \sqrt{V_{1} V_{2}} e^{i q_{3} z_{3}}-\frac{\Delta}{4 p}\left(\frac{V_{1}-V_{2}}{V_{1} V_{2}}\right) E_{1}^{*} E_{2}^{*},
$$

where

$$
\begin{aligned}
\Delta & =1+\frac{\left|H_{+}\right|^{2}}{1+Q}+\frac{\left|H_{-}\right|^{2}}{1-Q}-2 \cos (\beta) \mathcal{R} e\left(H_{+} H_{-}^{*} e^{i(\beta+2 \theta)}\right) \\
H_{ \pm}(z, t) & =e^{\left(-B_{ \pm}+i \chi_{ \pm}\right) z} e^{\frac{-2 V_{1} V_{2}}{V_{1}-V_{2}}(p-i k) t}, \\
\omega & =-2 k \frac{V_{1} V_{2}}{V_{1}-V_{2}}, \\
\chi_{ \pm} & =k\left(\frac{V_{1}+V_{2}}{V_{1}-V_{2}} \mp \frac{1}{Q}\right), \\
B_{ \pm} & =p\left(\frac{V_{1}+V_{2}}{V_{1}-V_{2}} \mp Q\right), \tan (\beta)=k /(p Q), \\
Q & \left.=\frac{1}{p} \sqrt{\frac{1}{2}\left[r+\sqrt{r^{2}+4 k^{2} p^{2}}\right.}\right], \\
r & =p^{2}-k^{2}-a^{2}, \\
q_{n} & =q\left(V_{n+1}-V_{n+2}\right), \\
z_{n} & =z+V_{n} t, n=1,2,3 \bmod (3) .
\end{aligned}
$$

It should be pointed out that the expressions (59) and (60) of waves $E_{1}$ and $E_{2}$ could also be given in the form (17), that is in terms of the position $\xi(t)$ and polarization vector $\hat{\alpha}(t)$ of the boomeron by applying the transformations (41) and (42) to the expression (17). However, the equivalent expressions (59) and (60) are used here since they are more suitable to our analysis. Figure 6 displays the analytical boomeron solution corresponding to numerical dynamics of unstable simulton of Fig. 4. It is worth noting that the above solution depends upon seven real
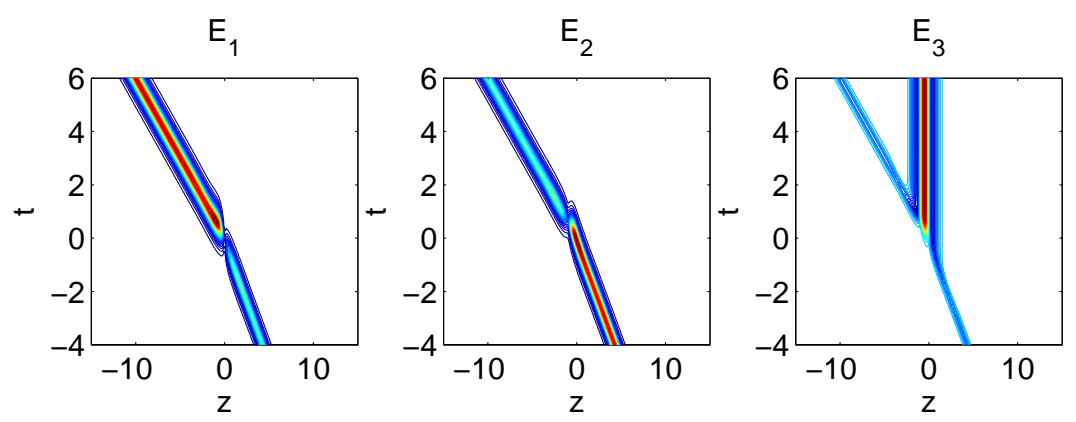

Fig. 6. Analytical boomeron solution describing the unstable TWRIS dynamics of Fig. 4. Parameters are $V_{1}=2, V_{2}=1, V_{3}=0, p=-1, a=1, k=0.5, q=1, \theta=\pi / 2$ (The triplet velocities are $V_{i}=1.1$ and $\left.V_{f}=1.68\left(V_{c r} \approx 1.3\right)\right)$.

parameters $V_{1}, V_{2}, p, k, q, \theta, a>0$. From the definition of $Q$, it is apparent that these parameters must be chosen in such a way that if $k=0$, then $p^{2}>a^{2}$. The analytical solution (59)-(61), while rather complicate at intermediate times, asymptotically consists of one or two coherent structures. In fact, let us consider first the decay process: if we assume $p<0$, for negative large $t(t \rightarrow-\infty)$ the boomeron is asymptotically composed of two bright pulses $\left(E_{1}, E_{2}\right)$ and a kink-like pulse $\left(E_{3}\right)$ traveling with the locked velocity $V_{i}$. If instead $t$ is large and positive $(t \rightarrow+\infty)$, the boomeron is composed of two bright pulses $\left(E_{1}, E_{2}\right)$ and a kink-like pulse $\left(E_{3}\right)$ traveling at the locked velocity $V_{f}\left(V_{f}>V_{i}\right)$, plus another pulse $\left(E_{3}\right)$ that travels with the 
linear group velocity $V_{3}$. The velocities $V_{f}$ and $V_{i}$ can be calculated from (59)-(61):

$$
\begin{gathered}
V_{i}=\frac{2 V_{1} V_{2}}{V_{1}+V_{2}-Q\left(V_{1}-V_{2}\right)} \\
V_{f}=\frac{2 V_{1} V_{2}}{V_{1}+V_{2}+Q\left(V_{1}-V_{2}\right)} .
\end{gathered}
$$

The triplet traveling at very large $|t|$ with the locked velocity $V_{i}\left(V_{f}\right)$ is in itself an exact solution of equations (40), namely it is the unstable (stable) TWRIS as presented in (48)-(50). Therefore, quite remarkably, the boomeron solution (59)-(61) provides an exact description of the decay from unstable to stable simultons. Let us consider next the situation where a stable TWRIS collides with an isolated pulse in the wave $E_{3}$, namely the excitation by absorption. Once again, this scattering process is exactly described by the boomeron solution (59)-(61), and it leads to the excitation of an unstable TWRIS, which is induced by the absorption of the isolated wave $E_{3}$. Indeed, whenever $p>0$ and $t$ is very large and negative, the boomeron (59)(61) is composed of a triplet consisting of two bright pulses (in waves $E_{1}, E_{2}$ ) and a kink-like pulse (in wave $E_{3}$ ), all traveling with the same velocity $V_{i}$, plus an isolated pulse in wave $\left(E_{3}\right)$ that travels with the linear group velocity $V_{3}$. The triplet and the isolated pulse collide and, as a result, the pulse in $E_{3}$ is completely absorbed by the triplet. Finally, for very large and positive $t$ the boomeron consists of a single triplet formed by two bright pulses (in waves $E_{1}, E_{2}$ ) and a kink-like pulse (in wave $\left.E_{3}\right)$, again traveling together with the velocity $V_{f}\left(V_{f}<V_{i}\right.$, see $(62)$ and $(63))$. Note that the asymptotic boomeron triplets traveling with velocities $V_{i}$ and $V_{f}$ can be analytically mapped into the stable and unstable TWRIS as given in (48)-(50). In conclusion, the analytical solution (59)-(61) with $p>0$ provides the exact description of the excitation of an unstable TWRIS as a result of the inelastic collision between a stable TWRIS and an isolated wave packet. Figure 7(a) displays the analytical boomeron solution corresponding to the collision between a stable TWRIS and a pulse in wave $E_{3}$. Whereas Fig. $7(\mathrm{~b})$ shows the inelastic scattering of the TWRIS and the isolated pulse as numerically computed by integrating the equations (40) with the initial data at $t=-5$ equal to the solution of Fig. $7(\mathrm{a})$. As it can be seen in Fig. 7(b), the excited unstable TWRIS has a finite lifetime since it eventually decays into a stable or ground state TWRIS via the emission of another isolated wave. It is worth noting that both excitation and decay processes may be fully described by properly adjusting the parameters of Eqs.(59)-(61). The dynamics of the scattering between TWRIS and isolated waves is analogous to the interaction between a two-level atom and radiation. Indeed, transitions between excited and ground TWRIS states are induced by the absorption and spontaneous emission of an isolated pulse in the wave $E_{3}$. Let us now briefly discuss the role of the various parameters in equations (59)-(61). Two of these parameters (i.e. the velocities $V_{1}$ and $V_{2}$ ) are fixed by the linear dispersive properties of the medium. We are thus left with five independent real parameters, namely $p, k, q, a, \theta$ (with the restrictions $a>0$ and $0 \leq \theta<2 \pi$ ). We would like to point out that, thanks to the above discussion, the specification of these parameters allows us to define all properties of both unstable and stable TWRIS. Indeed, these simultons result as asymptotic states of the boomeron expression (59)-(61) in the limit of $|t| \rightarrow \infty$. The parameter $p$ is associated with the rescaling of the wave amplitudes, and of the coordinates $z$ and $t$. Whereas $a$ measures the amplitude of the kink background in wave $E_{3}$. The value of $k$ is related to the soliton wave-number. The parameter $q$ simply adds a phase shift which is linear in both $z$ and $t$. Finally, $\theta$ fixes the shape of the stationary kink pulse $E_{3}$. By adjusting the various degrees of freedom of the boomeron family of solutions (59)-(61), one may foresee the dynamical reshaping of the amplitude, phase, and velocity of the TWRIS. Additionally, one may fully describe the process of energy exchange among the three interacting waves.

\subsection{Three-wave simulton collisions}

In order to emphasize the novel and striking features of the scattering between TWRIS and isolated waves, let us briefly consider collisions between two different TWRIS. We denote with 

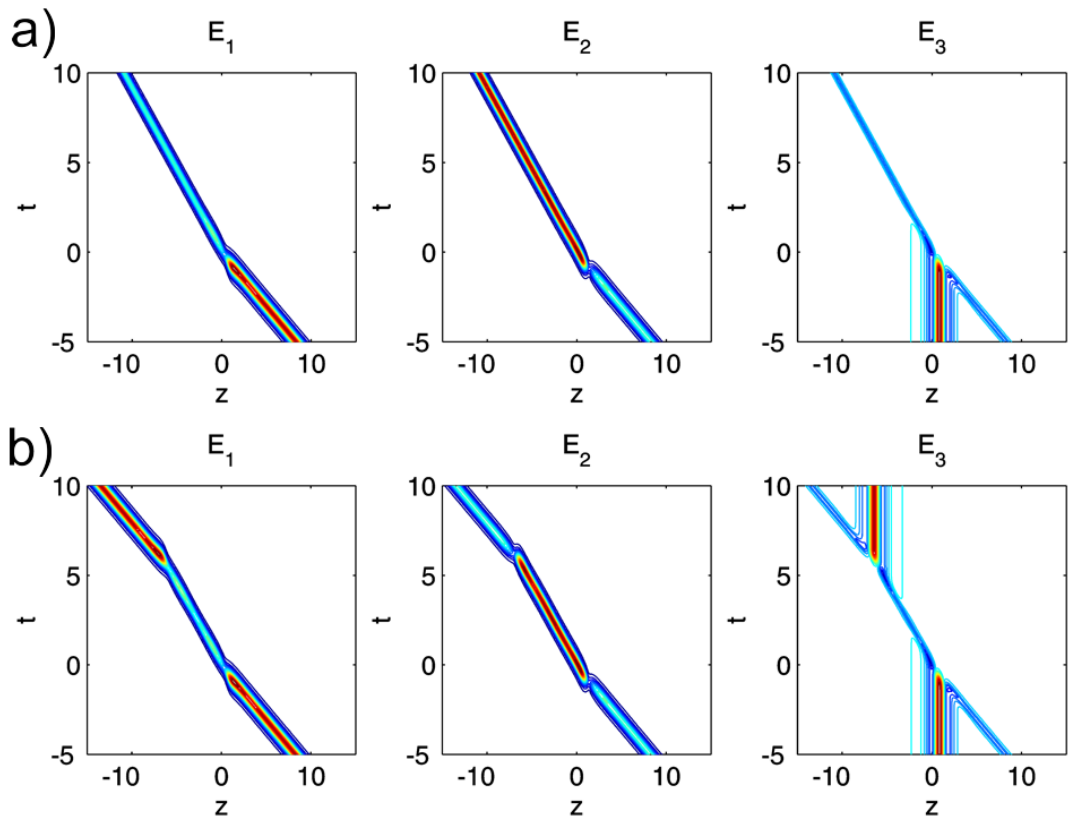

Fig. 7. a) Analytical boomeron solution describing the collision of a stable TWRIS with a single pulse in wave $E_{3}$. Parameters are $V_{1}=2, V_{2}=1, V_{3}=0, p=1, a=1, k=0.5, q=1, \theta=\pi / 6$. The triplet velocities are $V_{i}=1.68$ and $V_{f}=1.1\left(V_{c r} \approx 1.3\right)$. b) Numerical excitation-decay process.
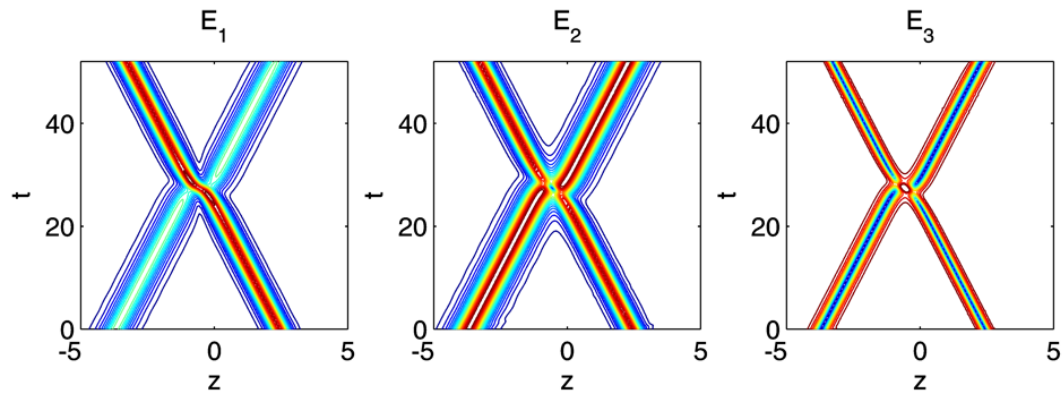

Fig. 8. Collision of two stable TWRIS with different velocities. Fast simulton $V=1.9$, slow simulton $V=1.7$. Simulation is performed in reference frame moving at velocity $V_{\text {ref }}=1.8$.

$u_{n}$ and $v_{n}(n=1,2,3)$ the three components of the first and the second simulton, respectively, both of them centered at $z=0$ for $t=0$. Moreover, we will consider the following initial condition (at $t=0)$

$$
\begin{aligned}
& E_{1}(z, 0)=u_{1}\left(z-\frac{z_{0}}{2}, 0\right)+v_{1}\left(z+\frac{z_{0}}{2}, 0\right) e^{i \alpha} \\
& E_{2}(z, 0)=u_{2}\left(z-\frac{z_{0}}{2}, 0\right)+v_{2}\left(z+\frac{z_{0}}{2}, 0\right) e^{-i \alpha}, \\
& E_{3}(z, 0)=u_{3}\left(z-\frac{z_{0}}{2}, 0\right) \times v_{3}\left(z+\frac{z_{0}}{2}, 0\right) /\left|u_{3}(\infty, 0)\right|,
\end{aligned}
$$

where $z_{0}$ is the initial separation between the simultons, and $\alpha$ is a constant phase factor. Since equations (40) are completely integrable, one may intuitively expect (but not give for granted, in view of the previously discussed absorption and decay phenomena) that interactions between two initially well-separated TWRIS do not modify the shapes of triplets that emerge 

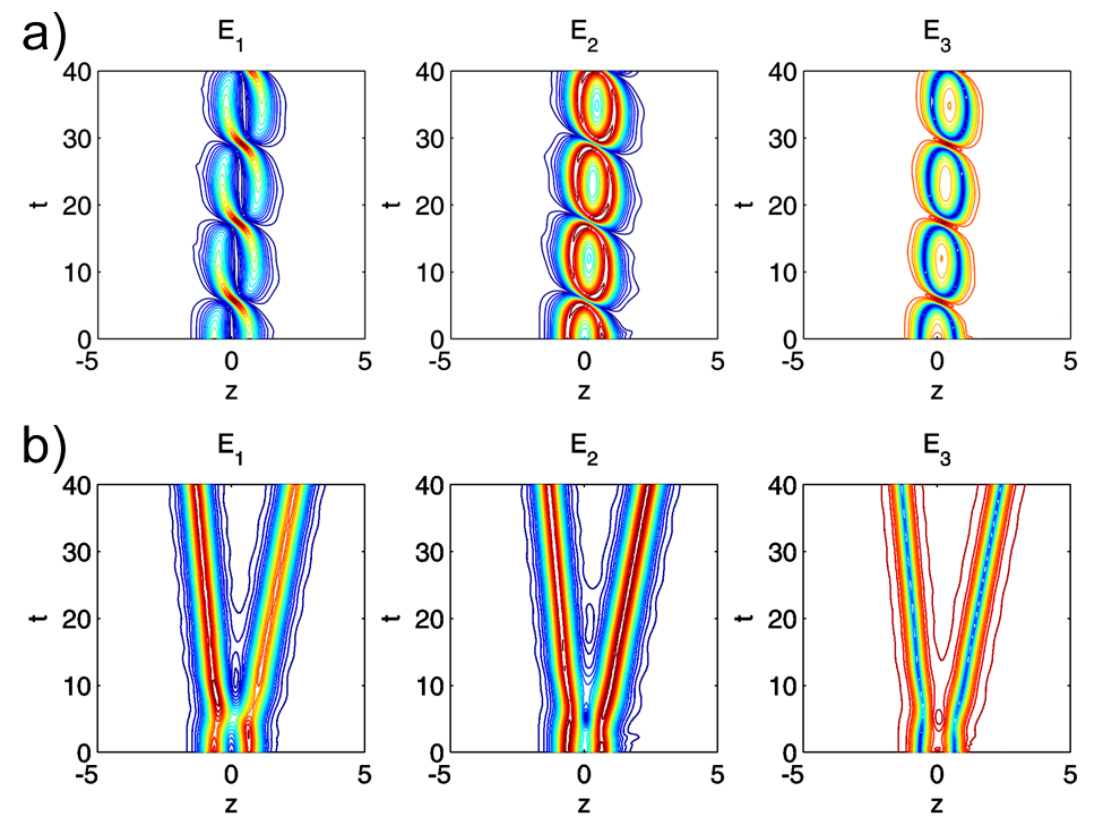

Fig. 9. a) Collision of two equal and in-phase stable TWRIS with the same velocity $V=1.8$; b) collision of two equal and $\pi$ out-of-phase stable TWRIS with the same velocity. Simulations are performed in reference frame moving at velocity $V_{\text {ref }}=1.8$.

after the collision. Indeed, the numerical simulation of Fig. 8 shows that two TWRIS with different velocities penetrate and cross each other with no change of their shapes. The only effect of the interaction is a spatial shift and a phase shift, as it happens with ordinary bright TWRI solitons [43]. However, in a manner similar to cubic nonlinear Schrödinger solitons ([44, 45], [46] and references therein), whenever the initial simulton separation is reduced, complex interaction phenomena may take place owing to the excitation of higher order soliton solutions. For example, Fig. 9(a) shows that two equal and in-phase $(\alpha=0)$ TWRIS with the same velocity attract each other and periodically collapse. Whereas Fig. 9(b) shows that a repulsive force exists between two equal and out-of-phase $(\alpha \neq 0)$ solitons with the same velocity. In this case, two distinct TWRIS moving with different velocities emerge from the initial collision. Hence TWRI solitons may cross, attract or repel each other depending on their initial separation, velocity difference, and relative phase.

\section{Conclusions and outlook}

Nonlinear wave coupling may give rise to novel soliton structures such as the boomerons, whose dynamics is far richer than that of standard, uncoupled wave solitons. In this work we have shown that this is certainly the case for parametric resonant three-wave coupling, a model which is of wide applicability in a variety of physical contexts (plasma physics, nonlinear optics, acoustics, fluid dynamics, and solid state physics). In nonlinear optics, we envisage that propagation effects related to three-wave boomerons should be observable at realistic intensity levels (of the order of $10 \mathrm{MW} / \mathrm{cm}^{2}$ ) when mixing picosecond light pulses in quadratic nonlinear materials. Indeed, it is already known that three-wave solitons may be exploited in order to enhance the efficiency of the frequency conversion of short optical pulses in the presence of group-velocity mismatch between the three waves $[24,25,43]$. Nevertheless, such process is hampered by the propagation instability of the up-converted pulse. This means that, for a given input pulse duration and intensity, a narrow range of tolerance results for the optimal nonlinear crystal length, thus limiting in practice the applicability of the soliton method. As we will discuss in separate 
reports, extended simultons and boomerons may provide a means to overcome this limitation: as it turns out, enhanced and stable parametric frequency conversion of short optical pulses may be achieved by mixing a short optical pulse with a quasi-continuous background pulse. As a further perspective, we believe that optical boomerons may open a new research direction in the growing field of slow and fast light, as they enable for the intensity-dependent and stable control of the speed of light pulses. Analytical solutions of the three-wave interaction equations (40) also point to the possibility of cavity-less short pulse train generation by means of the optical trappon effect (i.e., back and forth oscillations of self-trapped pulses inside a quadratic nonlinear medium, in the absence of any localized or distributed linearly reflecting mirror). We plan to investigate in the near future the possibility of generating boomeronic solutions in integrable stimulated scattering (i.e., Raman or Brillouin) three-wave equations, that is beyond the parametric mixing case of equations (40). As discussed in sections 1 and 2, we may anticipate that dynamically evolving solitons should exist and thus could be observed in a variety of nonlinear coupled mode evolution equations, including: parametric mixing in media with both quadratic and cubic nonlinearity, polarization evolution of short optical pulses in the presence of polarization mode dispersion and both instantaneous and relaxing nonlinearity (with a response time much longer than the pulse durations), etc. In conclusion, boomerons are definitely gaining the status of readily observable physical structures: their laboratory demonstration appears to be well at hand.

\section{References}

1. C. S. Gardner, J. M. Greene, M. D. Kruskal and R. M. Miura, Phys. Rev. Lett. 19, (1967) 1095.

2. E. Fermi, J. Pasta, S. Ulam, Studies of Nonlinear Problems, Document LA-1940 (1955).

3. A. Degasperis, M. Olshanetsky and A. M. Perelomov, Nuovo Cimento 29A, (1980) 245.

4. S. V. Manakov, Sov. Phys. JETP 38, (1974) 248.

5. F. Calogero and A. Degasperis, Lett. Nuovo Cimento 15, (1976) 65.

6. A. Degasperis, in: Nonlinear evolution equations solvable by the spectral transform, edited by F. Calogero (Pitman, London, 1978), 97.

7. F. Calogero and A. Degasperis, Lett. Nuovo Cimento 16, (1976) 425.

8. A. Degasperis, C. Rogers and W. Schief, Studies Appl. Math. 109, (2002) 39.

9. F. Calogero and A. Degasperis, Studies Appl. Math. 113, (2004) 91.

10. F. Calogero and A. Degasperis, J. Phys. A: Math. Gen. 39, (2006) 8349.

11. A. Degasperis and S. Lombardo, J. Phys. A: Math. Theor. 40, (2007) 961.

12. A. Degasperis and S. Lombardo, in preparation.

13. V. E. Zakharov and S. V. Manakov, JETP Lett. 18, (1973) 243.

14. V. E. Zakharov and S. V. Manakov, Sov. Phys. JETP. 42, (1976) 842.

15. F. Calogero and A. Degasperis, Physica D 200, (2005) 242.

16. J. A. Armstrong, S. S. Jha, and N. S. Shiren, IEEE J. Quantum Electron. QE-6, (1970) 123.

17. K. Nozaki and T. Taniuti, J. Phys. Soc. Jpn. 34, (1973) 796.

18. Y. Ohsawa, K. Nozaki, J. Phys. Soc. Jpn., 36, (1974) 591.

19. K. Nozaki, J. Phys. Soc. Jpn., 37, (1974) 1124.

20. D. J. Kaup, Stud. Appl. Math. 55, (1976) 9.

21. A. Bers, D. J. Kaup, and A. H. Reiman, Phys. Rev. Lett. 37, (1976) 182.

22. A. H. Reiman, A. Bers, and D. J. Kaup, Phys. Rev. Lett. 39, (1977) 245.

23. Y. N. Taranenko, and L. G. Kazovsky, IEEE Phot. Tech. Lett. 4, (1992) 494.

24. E. Ibragimov and A. Struthers, Opt. Lett. 21, (1996) 1582.

25. E. Ibragimov, A. Struthers, J. Opt. Soc. Am. B, 15, (1998) 97.

26. A. Picozzi and M. Haelterman, Opt. Lett. 23, (1998) 1808.

27. A. Picozzi and M. Haelterman, Phys. Rev. E 59, (1999) 3749.

28. D. J. Kaup, A. Reiman, and A. Bers, Rev. Mod. Phys., 51, (1979) 275.

29. V. M. Malkin, G. Shvets, and N. J. Fish, Phys. Rev. Lett. 84, (2000) 1208.

30. I. Y. Dodin and N. J. Fish, Phys. Rev. Lett. 88, (2002) 165001.

31. A. Degasperis and S. Lombardo, Physica D 214, (2006) 157.

32. A. Degasperis, M. Conforti, F. Baronio and S. Wabnitz, Phys. Rev. Lett. 97, (2006) 093901.

33. M. Conforti, F. Baronio, A. Degasperis, and S. Wabnitz, Phys. Rev. E 75, (2007) 065602(R). 
34. A. V. Buryak et. al, Phys. Rep. 370, (2002) 63.

35. M. Conforti, A. Locatelli, C. De Angelis, A. Parini, G. Bellanca, and S. Trillo, J. Opt. Soc. Am. B 22, (2005) 2178 .

36. D. E. Pelinovsky, A. V. Buryak, and Y. S. Kivshar, Phys. Rev. Lett. 75, (1995) 591.

37. A. V. Buryak, Y. S. Kivshar, and S. Trillo, Phys. Rev. Lett. 77, (1996) 5210.

38. D. Mihalache, D. Mazilu, L. C. Crasovan, and L. Torner, Phys. Rev. E 56, (1997) R6294.

39. D. Mihalache, D. Mazilu, and L. Torner, Phys. Rev. Lett. 81, (1998) 4353.

40. D. Mihalache, D. Mazilu, B. A. Malomed, and L. Torner, Opt. Comm. 169, (1999) 341.

41. Y. S. Kivshar and W. Królikovski, Opt. Lett. 20, (1995) 1527.

42. L. Torner, D. Mihalache, D. Mazilu, M. Santos, and N. N. Akhmediev, J. Opt. Soc. Am. B 15, (1998) 1476 .

43. E. Ibragimov and A. Struthers, J. Opt. Soc. Am. B, 14, (1997) 1472.

44. P. L. Chu and C. Desem, Electron. Lett. 21, (1985) 1133.

45. Y. Kodama and K. Nozaki, Soliton interaction in optical fibers, Opt. Lett. 12, (1987) 1038.

46. A. Hasegawa and Y. Kodama, Solitons in Optical Communications, Oxford University Press (1995). 